Artículo

\title{
Calibración de un prototipo para realizar mediciones continúas de radiación fotosintéticamente activa en sorgo
}

\author{
Gilbert Fresh López López ${ }^{1}$ \\ Diego Cruz Hernandez ${ }^{2}$ \\ Pedro Andrade Sanchez ${ }^{3}$ \\ Santos Gabriel Campos Magaña ${ }^{1}$ \\ Martin Cadena Zapata ${ }^{1}$ \\ Juan Antonio López López ${ }^{1 \S}$
}

${ }^{1}$ Universidad Autónoma Agraria Antonio Narro-Departamento de Maquinaria Agrícola. Calzada Antonio Narro núm. 1923, Buenavista, Saltillo, Coahuila, México. CP. 25315. (gilbert.flopezl@uaaan.edu.mx; camposmsg@hotmail.com; martincadenaz@gmail.com; juan.alopezl@uaaan.edu.mx). ${ }^{2}$ Postgrado en Ingeniería de Sistemas de Producción-Departamento de Maquinaria Agrícola-Universidad Autónoma Agraria Antonio Narro. Calzada Antonio Narro núm. 1923, Buenavista, Saltillo, Coahuila, México. CP. 25315. (zynchd@hotmail.com). ${ }^{3}$ Universidad de Arizona-Departamento de Ingeniería Agrícola y de Biosistemas. Tucson, Arizona. (pcandradesanchez@gmail.com).

${ }^{\S}$ Autor para correspondencia: juan.alopezl@uaaan.edu.mx.

\section{Resumen}

El presente trabajo tuvo como objetivo calibrar el funcionamiento de un prototipo para medición continua de radiación fotosintéticamente activa (PAR) y validar con pruebas de campo en el cultivo de sorgo (Sorghum spp.) en etapa final de su desarrollo. El prototipo desarrollado se basa en dos sondas de 80 sensores (fotodiodos). Para efectos de calibración se comparó la salida de este prototipo con un sensor comercial AccuPAR modelo LP-80 PAR/LAI Ceptometer (Empresa Decagon), dicha calibración se explica por la pendiente de un modelo de regresión lineal. Los resultados obtenidos fueron una pendiente de $6.22963 \mu \mathrm{mol} \mathrm{m}^{-2} \mathrm{~s}^{-1}\left(\mathrm{mV} \times 10^{2}\right)^{-1}$ con un coeficiente de determinación de 0.997, cumpliendo con los supuestos estadísticos al 95\%. Además, se observan el mapa de la variabilidad en el campo cuyos valores oscilan entre $\leq 794.4$ a 2160 $\mu \mathrm{mol} \mathrm{m} \mathrm{m}^{-2} \mathrm{~s}^{-1}$ indicando el límite inferior que existe mayor follaje y límite superior menor follaje. A partir de los datos generados por el prototipo se observó similitudes con el sensor comercial, lo cual sugiere que dicho prototipo recolecta los datos en menor tiempo y con mayor cobertura de muestreo, aprovechando la ventana de oportunidad del ciclo diurno de radiación solar.

Palabras claves: agricultura de precisión, ecuación de calibración, prototipo PAR.

Recibido: abril de 2021

Aceptado: junio de 2021 


\section{Introducción}

La tasa de fotosíntesis de una planta está relacionada a la cantidad de radiación fotosintéticamente activa (PAR) por sus siglas en inglés que es absorbida por la planta (McCree, 1981). Debajo de una canopia de planta los niveles de radiación pueden variar desde pleno sol a casi cero sobre el espacio de pocos centímetros. Por lo tanto, para mediciones confiables de PAR se requiere de muchas muestras en diferentes ubicaciones bajo la canopia (Decagon, 2016).

La PAR es necesaria para una entrada en aplicaciones comerciales como en fisiología de plantas, producción de biomasa e iluminación natural en un invernadero. Desafortunadamente la medición de PAR no es una práctica común establecida, actualmente se calcula con una constante de proporción del ancho de banda de radiación solar (Alados et al., 1996). Campbell (1981) mencionó que debido a que transfiere radiación de energía, es importante para las plantas por al menos tres razones: 1) la tasa fotosintética de una planta está relacionada a la cantidad de PAR absorbida por la planta; 2) la temperatura de una planta es en parte determinada por la tasa de absorción y emisión de radiación; y 3) la respuesta foto morfológica de la planta está determinada por la absorción de energía radiante en banda de frecuencia específica.

Especificaciones adecuadas de las propiedades de radiación ambiental incluyendo en cada uno de estos procesos requeridos en conocimiento de ambos la densidad de flujo radiante y distribución de banda de frecuencia de la radiación, ya que la variación espectral varía la fotosíntesis con la longitud de banda. La respuesta fotosintética por fotón absorbido no será independiente del espectro de la fuente de luz (Erol y Akdeniz, 1996; Grignetti et al., 1997; Pax-Lenney y Woodcock, 1997; Haboudane et al., 2002).

La densidad de flujo del fotón en la banda de frecuencia 400 a $700 \mathrm{~nm}$ puede ser considerada una medida adecuada de radiación PAR definida por estudios de Gaastra (1959); Ross (1975) la ejecución de mediciones de PAR involucra errores adicionales, los cuales pueden ser esperados y comparados en magnitud. Así, en la medición de PAR es improbable exceder una exactitud general de $\pm 10 \%$. Incluso este nivel de exactitud no puede ser alcanzado sin un buen cuidado en el diseño, calibración y uso de los instrumentos (Decagon, 2016; Zhou et al., 2016).

Se debe notar que PAR es una medición de la radiación disponible para la fotosíntesis y no debería ser tratada como una medición universal de la radiación disponible para el crecimiento. Desafortunadamente no existe tal medida. La radiación afecta a diferentes procesos de crecimiento cada una de los cuales debe tratarse independientemente (McCree, 1973; Shibles, 1976; Dybing, 1977). Viña y Gitelson (2005) mencionan que la cuantificación de las propiedades biofísicas de la vegetación terrestre y su variación en el tiempo son importantes para una evaluación rápida y precisa del estado de la vegetación y sus respuestas a las condiciones ambientales cambiantes.

Una de estas características biofísicas es la fracción de PAR absorbida. Los datos y las técnicas de los sensores remotos ya han demostrado ser relevantes para muchos requerimientos de inventario y monitoreo de cultivos. Diferentes estudios y experimentos demostraron su utilidad y viabilidad para abordar diversas cuestiones agrícolas, como la clasificación de cultivos y el mapeo de ellos. El propósito de esta investigación es generar la primera ecuación de calibración para un prototipo de medición de radiación fotosintéticamente activa (PAR) en tiempo real ajustado a un modelo de predicción. 


\section{Materiales y métodos}

El desarrollo de este proyecto de investigación se realizó en las instalaciones de la estación experimental Maricopa Agricultural Center de la Universidad de Arizona en Maricopa, AZ, USA, en el periodo de invierno de 2016 y consistió en el diseño y construcción de un prototipo para recolección de datos de la radiación fotosintéticamente activa (PAR) por sus siglas en inglés en continuo movimiento en un cultivo de sorgo.

El diseño conceptual del prototipo se presenta en la Figura 1, que se integra por: 1) perfil estructural de aluminio para un ensamble rápido; 2) rueda giratoria de tipo cojinete para poder maniobrar; 3 ) dos sondas tipo LP-80, cada una cuenta con 80 fotodiodos conectados de forma paralela obteniendo 8 secciones y una resistencia de $420 \Omega$ para captar la señal; 4) un GPS StarFire300 John Deere que cuenta con la facilidad de conexión compatible con el sistema de adquisición mediante la conexión serial RS-232; 5) un sistema de adquisición de datos Micrologger CR3000 (Campbell Scientific Company, USA); 6) dos botones para iniciar y pausar la adquisición de datos; y 7) batería de 12V para alimentación.

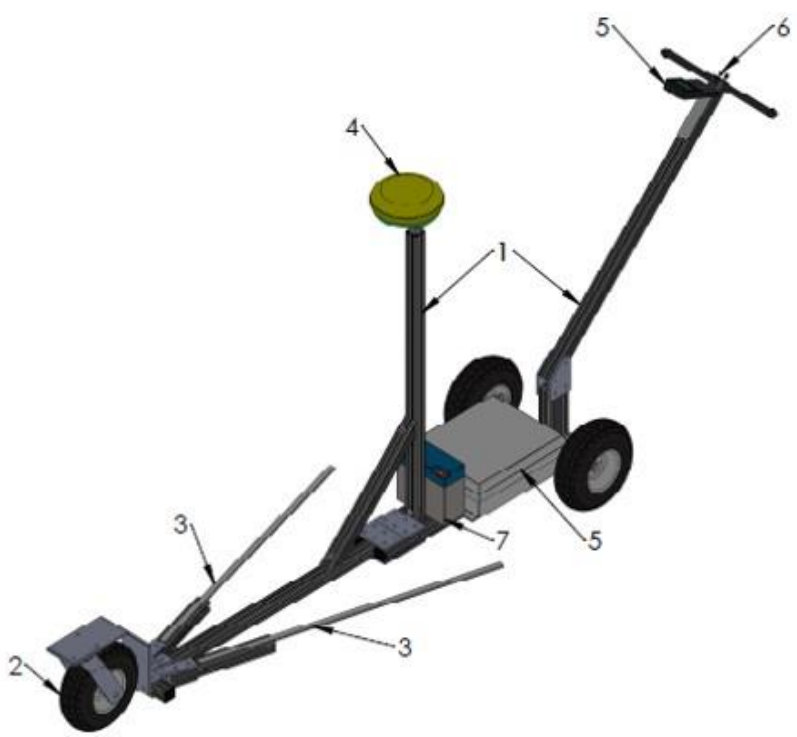

\section{Figura 1. Diseño conceptual del prototipo.}

\section{Primera ecuación de calibración}

\section{Adquisición de datos}

En el campo F13 ubicado en $111^{\circ} 58^{\prime} 29.70^{\prime \prime}$ longitud oeste, $33^{\circ} 4^{\prime} 31.40^{\prime \prime}$ latitud norte a una altitud de $360 \mathrm{~m}$. Para la adquisición de datos del prototipo se codifico un programa de control en el software CRBasic Editor, el cual conto con 16 secciones de forma alterna para la señal analógica (voltaje) de los sensores, localización de GPS. Los botones de inicio-pausa y velocidad de adquisición de datos a $5 \mathrm{~Hz}$. 
El programa de control se grabó en el CPU del sistema de adquisición. Se registró de forma continua en un rango de tiempo de doce horas los datos de los sensores. Por otro lado, el sensor manual AccuPAR modelo LP-80 PAR/LAI Ceptometer se utilizó para la medición manual tomando valores promedio de diez lecturas por cada hora durante toda la medición del prototipo (Figura 2).

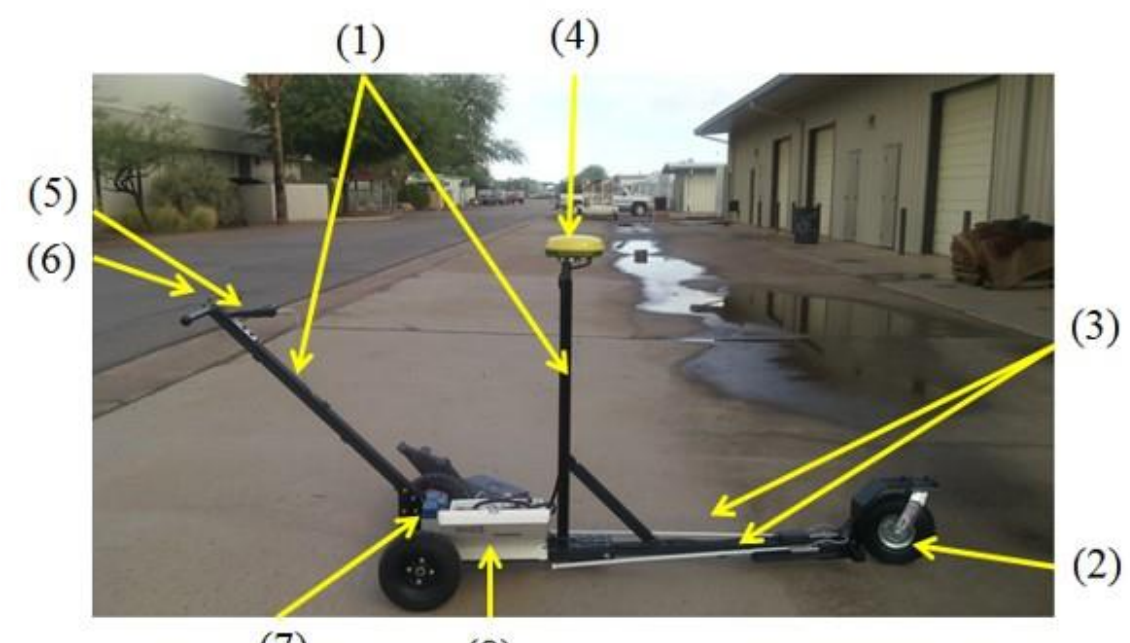

(7)

(8)

Figura 2. Elementos que integran el prototipo. 1) chasis del prototipo; 2) rueda controladora; 3) sensores; 4) GPS; 5) extensión de control; 6) botones encendido-pausa; 7) batería de 12 V; y 8) micrologger CR3000.

\section{Ecuación de calibración}

$\mathrm{y} i=\beta_{0}+\beta_{1} \mathrm{x}_{\mathrm{i}}+\varepsilon_{\mathrm{i}}$ para generar la ecuación de calibración se ajustan los datos del prototipo y sensor AcuPAR Ceptometer modelo LP-80 (manual). Para el análisis en el software R v. 3.3.3 con un modelo de regresión lineal (ecuación 1). Se sumaron los promedios de los voltajes de cada hora de las ocho secciones del sensor para comparar con el promedio del sensor manual por cada hora y comparar los supuestos estadísticos para la confiabilidad de este, estas pruebas son: normalidad (Shapiro-Wilk), independencia de los errores (Durbin-Watson y Box-Ljung) y heterocedasticidad (Breusch-Pagan). Donde: $\mathrm{y}_{\mathrm{i}}=$ variable independiente (volts); $\mathrm{x}_{\mathrm{i}}=$ radiación fotosintéticamente activa $\left(\mu \mathrm{mol} \mathrm{m} \mathrm{m}^{-2} \mathrm{~s}^{-1}\right), \beta_{0}$ y $\beta_{1}=$ ordenada y pendiente, respectivamente; $\varepsilon_{\mathrm{i}}=$ error experimental.

\section{Pruebas en campo}

Para la validación del primer acercamiento de calibración se realizaron pruebas dentro del cultivo de sorgo en su etapa final de desarrollo, establecido en el campo F13. Se realizaron pruebas estáticas en sitios para comparar el comportamiento. Para las pruebas dinámicas, se realizaron recorridos de norte a sur en la ventana de oportunidad, considerando el mejor ángulo de la posición del sol con respecto al cultivo. Y con ello generar mapas de caracterización de la variable PAR con el prototipo. 


\section{Resultados y discusión}

En la Figura 2 se presenta el prototipo armado con las componentes de acuerdo con el diseño conceptual desarrollado. Para los sensores del prototipo se presentan dos gráficos medidos en voltajes en la Figura 3, los cuales describen el comportamiento de las mediciones en volts para la calibración durante $12 \mathrm{~h}$, también se presenta error debido a la posición del sol cuando sale y se oculta, haciendo sombra hacia los sensores.

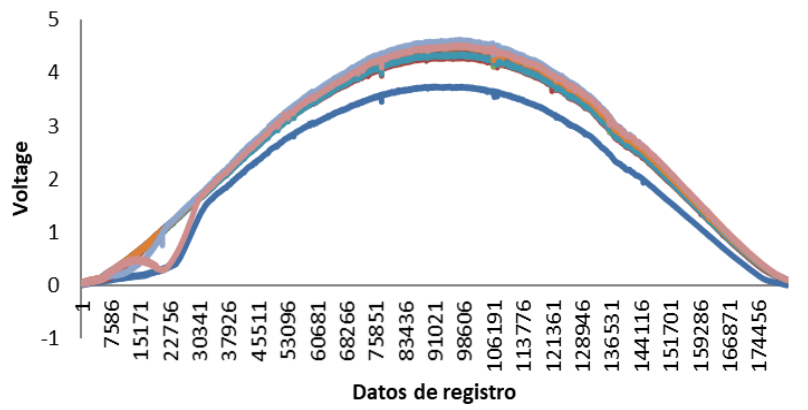

(a)

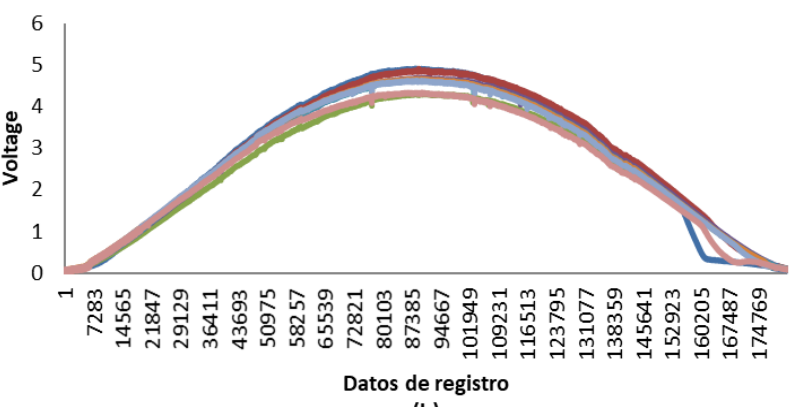

(b)

$\longrightarrow \mathrm{R} 01-\mathrm{R} 03-\mathrm{R} 05-\mathrm{R} 07-\mathrm{R} 09-\mathrm{R} 11-\mathrm{R} 13-\mathrm{R} 15$

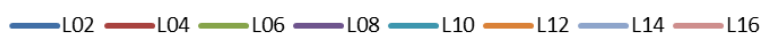

Figura 3. Lecturas de los sensores del prototipo del lado a) derecho; y b) izquierdo.

En la Figura 4 se muestran los datos del sensor manual tomada cada hora las unidades del sensor están dadas en micro mol por cada metro cuadrado por cada segundo $\left(\mu \mathrm{mol} \mathrm{m} \mathrm{m}^{-2} \mathrm{~s}^{-1}\right)$.

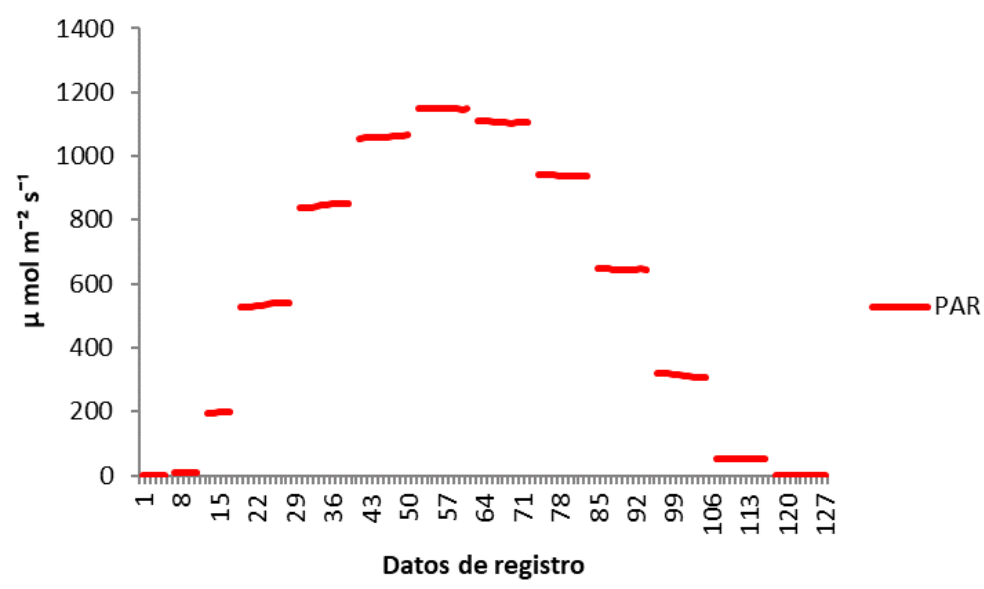

Figura 4. Datos con sensor manual AccuPAR LP80.

\section{Ecuación de calibración}

Se obtuvo la ecuación de calibración $6.22963 \mu \mathrm{mol} \mathrm{m}^{-2} \mathrm{~s}^{-1}\left(\mathrm{mV}^{-1} \times 10^{2}\right)^{-1}$ descrita por la pendiente del modelo de regresión lineal, la dispersión y tendencia de los datos se observa en la Figura 5. 


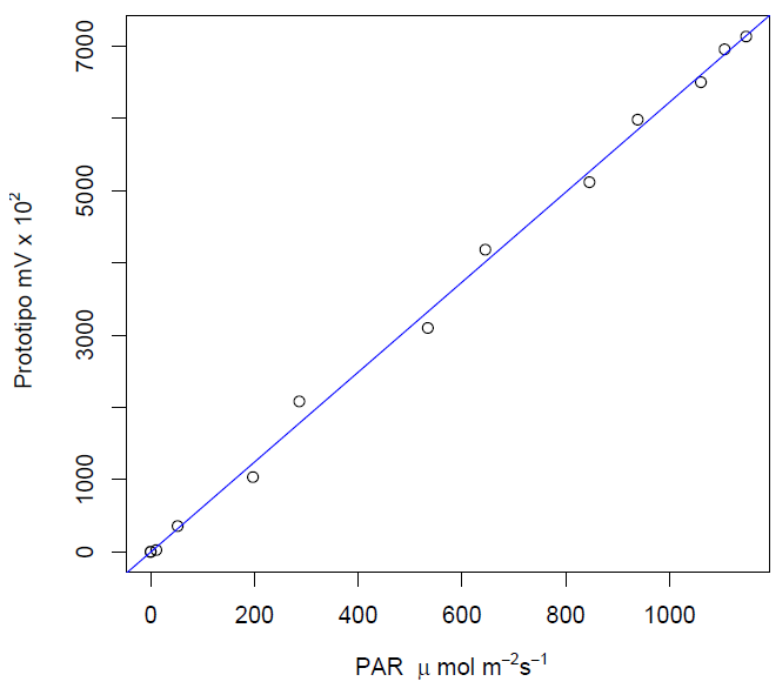

Figura 5. Diagrama de dispersión y tendencia de los datos.

Mientras que en el análisis se observa en el Cuadro 1, que la pendiente tiene alta significancia y el coeficiente de determinación $\left(\mathrm{R}^{2}\right)$ de 0.9973 esto representa alta confiabilidad del modelo por lo tanto alta confiabilidad de la ecuación.

Cuadro 1. Análisis de modelos de regresión lineal.

\begin{tabular}{ccccc}
\hline Coeficientes & Estimación & Error estándar & Valor t & $\operatorname{Pr}(>|\mathrm{t}|)$ \\
\hline Intercepto & -2.61578 & 67.0953 & -0.039 & 0.97 \\
RR & 6.22963 & 0.09806 & 63.526 & $1.82 \mathrm{e}-15^{* * *}$ \\
& Error estándar de residual: 155.2 en 11 grados de libertad & \\
& R-cuadrada múltiple: 0.9973, R-cuadrada ajustada: 0.997 & \\
& F-estadística: 4036 en 1 y 11 GL, $p$-valor: 1.822 e-15 & \\
\hline
\end{tabular}

Código de significância $={ }^{* * *}$ para $p \leq 0.001$.

El modelo cumple los supuestos estadísticos al 95\% indicando que los residuales tienen una distribución normal, independencia de residuales y varianzas independientes, esto indica que el método de análisis es confiable sin sufrir algún sesgo.

\section{Pruebas en campo}

En la Figura 6 se observan las pruebas de forma estática comparando el sensor manual LP80 (línea azul) y el prototipo obteniendo el voltaje y multiplicando por la ecuación de calibración llamando 'PAR estimado' (línea roja) la tendencia de los equipos similares, obteniendo un error promedio de $5 \%$. 


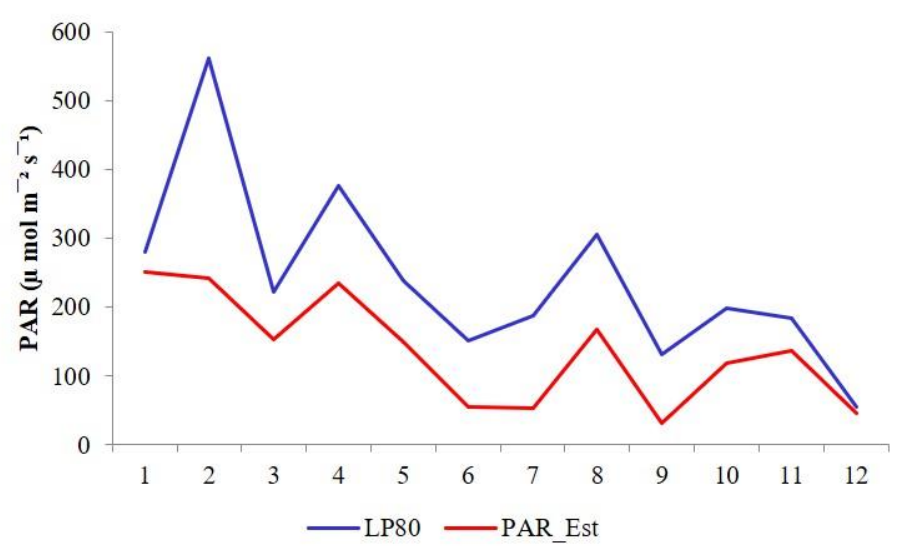

Figura 6. Gráfico de pruebas de PAR estáticas en campo.

En las pruebas estáticas se observa el mapa de caracterización del PAR teniendo mayor cobertura en campo en menor tiempo de muestreo y esto lo hace más eficiente debido a la ventana de oportunidad del sol. A continuación, se presentan el mapa de la prueba dinámica en donde se observa la variabilidad del sitio. En la Figura 7 se observó el mapa con una escala de colores en donde el color más claro representa el registro de poco PAR esto indica que en esa área el follaje de la planta tiene mayor cobertura y las áreas de color intenso representan que se tuvo mayor registro lo que indica que las plantas contaban con menor follaje.

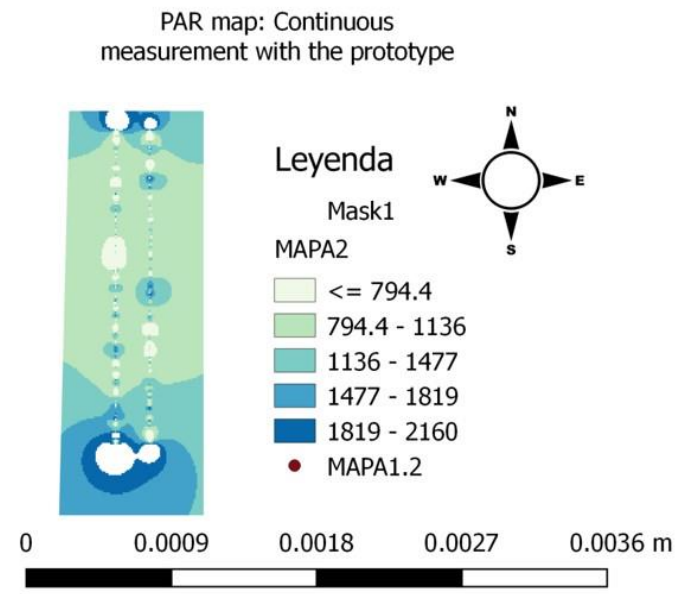

Figura 7. Mapa de caracterización de PAR.

La radiación fotosintéticamente activa se encuentra en 400 a 700 nanómetros de ancho de banda. Esto representa la porción del espectro solar que las plantas utilizan para la fotosíntesis. Bajo un dosel de la planta, los niveles de radiación pueden variar desde altos a bajos niveles sobre el espacio de unos pocos centímetros. Por lo tanto, para mayor confiabilidad de la medición de PAR requiere muchas muestras en diferentes ubicaciones bajo el dosel y sitios del campo (Decagon, 2016).

Andrade et al. (2004) mencionan que se ha incrementado el interés en el uso de sensores para la obtención de información de las variables del suelo y de los cultivos, muchos de estos sensores están integrados con un GPS para obtener los mapas de las diferentes variables debido a que con mayor densidad de datos se pueden hacer mejores cálculos para la toma de decisiones. El 
coeficiente de determinación del prototipo obtenido durante la calibración muestra alta confiabilidad para ser utilizado en campo y obtener más información sobre la radiación fotosintéticamente activa en menor tiempo.

\section{Conclusiones}

Se obtuvo la primera ecuación de calibración para el prototipo de medición de radiación fotosintéticamente activa (PAR) con un coeficiente de 0.9973 que representa confiabilidad de muestreo y un error promedio de 5\% en comparación con el sensor manual AccuPAR LP-80.

\section{Literatura citada}

Andrade, S. P.; Upadhyaya, S. K.; Jenkins, B. M.; Plouffe, C. and Poutre, B. 2004. Field evaluation of the improved version of the UCDavis compaction profile sensor (UCD CPS). Paper number 041037. ASAE Annual. Meeting. Doi:10.13031/2013.17644.

Alados, I.; Foyo-Moreno, I. and Alados-Arboledas, L. 1996. Photosynthetically active radiation: measurements and modelling. Agricultural and forest meteorology. 78(1996):121-131. Doi: 0168-1923(95)02245-7.

Decagon-Device, I. 2016. Accupar PAR/LAI Ceptometer model LP-80, operation's manual. http://library.metergroup.com/Manuals/10242_Accupar\%20LP80_Web.pdf. 1-82 p.

Dybing, C. D. and Lay, C. 1981. Yields and yield components of flax, soybean, wheat, and oats treated with morphactins and other growth regulators for senescence delay 1. Crop Science. 21(6):904-908. https://doi.org/10.2135/cropsci1981.0011183X002100060025x.

Erol, H. and Akdeniz, F. 1996. A multispectral classification algorithm for classifying parcels in an agricultural region. Inter. J. Remote Sensing. 17(17):3357-3371. Doi: 01431169608949156.

Gaastra, P. 1959. Photosynthesis of crop plants as influenced by light, carbon dioxide, temperature and stomatal diffusion resistance. Meded Landbouwhogesch Wageningen. 59(13):1-68.

Haboudane, D.; Miller, J. R.; Tremblay, N.; Zarco-Tejada, P. J. and Dextraze, L. 2002. Integrated narrow-band vegetation indices for prediction of crop chlorophyll content for application to precision agriculture. Remote Sensing Environ. 81(2):416-426. Doi: 10.1016/S00344257(02)00018-4.

Mc-Cree, K. J. 1973. A rational approach to light measurement in plant ecology. Curr. Adv. plant sci. 3(4):39-43.

Mc-Cree, J. K. 1981. Photosynthetically active radiation. Physiological plant ecology I. Springerverlag, Berlin. 12(01):41-55.

Pax-Lenney, M. y Woodcock, C. E. 1997. The effect of spatial resolution on the ability to monitor the status of agricultural lands. Remote Sensing Environ. 61(2):210-220. Doi: 10.1016/S0034-4257(97)00003-5.

Ross, J. 1975. Radiative transfer in plant communities. Monteith, J. (Ed.). vegetation and the atmosphere. Vol 1. Academic press. New York. 13-55 pp.

Shibles, R. 1976. Terminology relating to photosynthesis. Crop Sci. 16(3):437-439.

Viña, A. and Gitelson, A. A. 2005. New developments in the remote estimation of the fraction of absorbed photosynthetically active radiation in crops. Geophysical Research Letters. 32(17):1-4. Doi: 10.1029/2005GL023647.

Zhou, J.; Khot, L. R.; Bahlol, H. Y.; Boydston, R. and Miklas, P. N. 2016. Evaluation of ground, proximal and aerial remote sensing technologies for crop stress monitoring. IFACPapersOnLine. 49(16):22-26. Doi: 10.1016/j.ifacol.2016.10.005. 